\title{
The influence of emotional valence on word recognition in people with aphasia
}

\author{
Caroline Newton, Helena Thornley \& Carolyn Bruce \\ Language \& Cognition, Division of Psychology \& Language Sciences, University College London, UK
}

\section{Introduction}

People with aphasia frequently have difficulties in understanding and producing single words, with performance affected by a range of psycholinguistic variables, including word length and frequency. This study considers the effects on lexical processing of using emotionally valenced words: words that evoke an emotion but do not directly label one (e.g. negative valence $=$ poison, positive valence $=$ peace). Studies of neurotypical adults have found that emotional valence may also affect lexical processing efficiency (Kousta, Vinson \& Vigliocco, 2009). It is possible that emotional valence is therefore an additional psycholinguistic variable that influences performance for people with aphasia, with implications for assessment and materials used in intervention.

The current study therefore aimed to investigate the effects of emotional valence on single word processing in people with aphasia. People with and without aphasia completed written lexical decision task, in which emotional valence was manipulated.

\section{Methods}

\section{Participants}

Forty people took part in this study, 20 people with chronic aphasia (age range: $36-83$ ) and 20 age, education and gender matched neurotypical controls. Both groups comprised 10 men and 10 women. The participants with aphasia were recruited from a community clinic in London and were at least six months post-stroke. This group had differing communication difficulties, with varying levels of severity as assessed by the Western Aphasia Battery (Kertesz, 2006). The majority of participants presented with anomic aphasia (15/20) and Aphasia Quotient scores indicated severity ranging from $46 \%$ to $96.8 \%$.

\section{Stimuli}

Stimuli were adapted from those used by Kousta at al. (2009). There were 228 items in total:

- 114 words: 38 negative, 38 positive and 38 neutral words which were matched in terms of emotional valence, arousal, concreteness, imageability, age of acquisition, familiarity, frequency, orthographic neighbourhood, number of letters, syllables and morpheme and mean positional bigram frequency.

- 114 non-words: 38 additional negative, positive and neutral words were also selected, which were matched to pair with another word by length. One letter was then changed to create nonwords that were pronounceable and orthographically licit. 
Two items and their associated pairs from the original set used by Kousta et al (2009) were excluded from the current item set because they provoked responses below chance level in the previous study.

\section{Procedure}

Participants were tested individually, and the lexical decision task was presented via computer. At the start of each trial, a fixation cross appeared in the centre of the screen for $800 \mathrm{~ms}$, followed by a letter string (either a word or a non-word) with a tick and cross symbol which remained on screen until participants made their response. No time limit was set for registering a response as it was anticipated that the group with aphasia would require longer to respond. There was a blank intertrial interval of $1000 \mathrm{~ms}$. The stimuli were presented in a randomised order and participants were unable to self-correct errors. The computer program used to present the stimuli recorded response times and response accuracy per letter string.

\section{Results}

In order to remove the influence of anticipatory responses or attention lapses for reaction time data, incorrect responses, responses below $150 \mathrm{~ms}$ and responses that deviated above 3 standard deviations from the mean were excluded from analysis. Mixed ANOVAs were used to explore the effects of group and valence on accuracy and response time in the lexical decision task.

For accuracy, the ANOVA revealed that those with aphasia performed significantly less accurately than control participants. Across the whole group, accuracy was significantly higher for words with positive valence than for negative and neutral valence. A significant interaction was found between emotional valence and group, which revealed that participants with aphasia scored significantly more accurately for words with positive valence, but for the controls no significant differences were found between valence types (see Figure 1). 


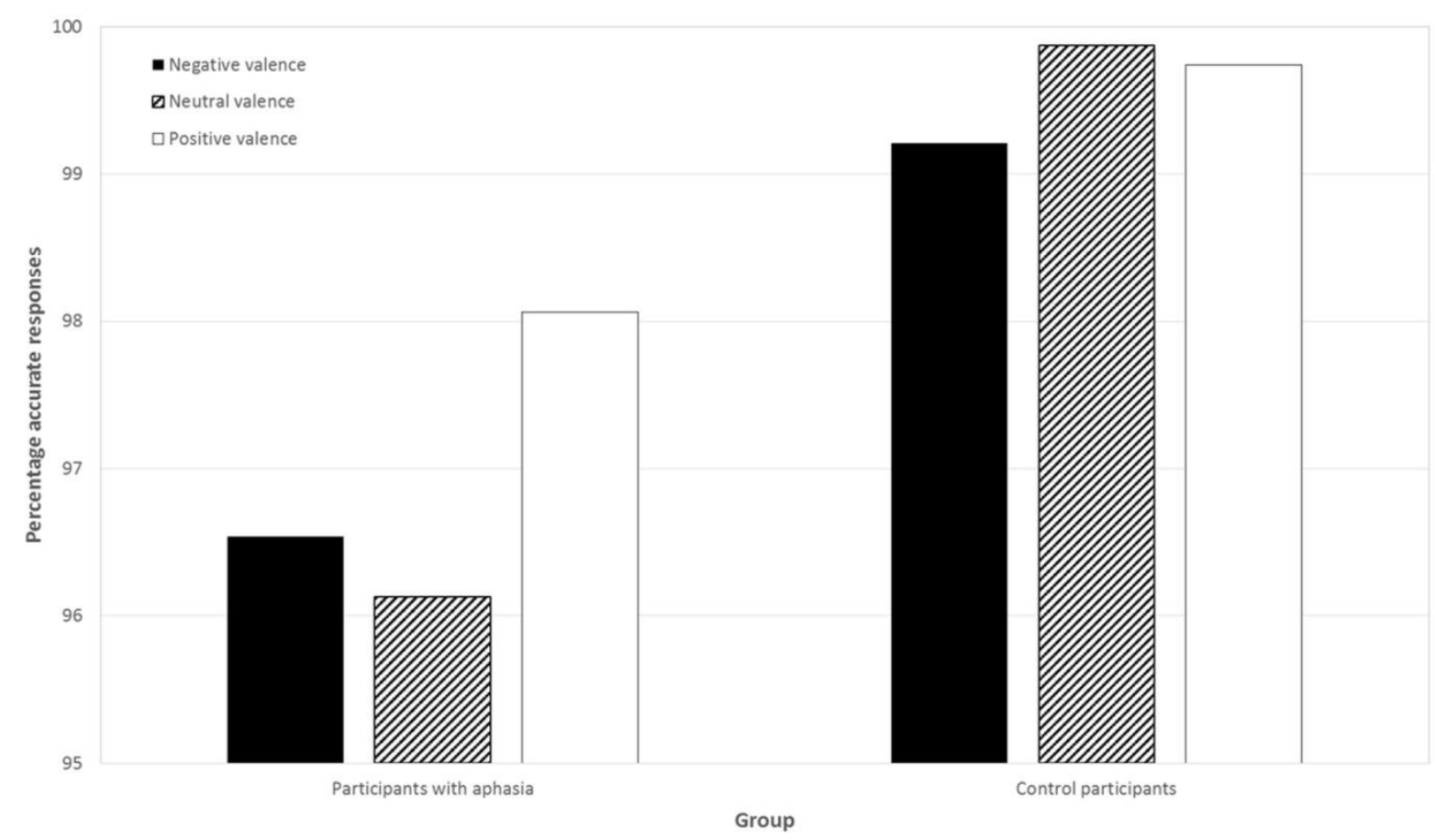

Figure 1. Accuracy of performance for participants with and without aphasia.

The second ANOVA exploring response time revealed that the participants with aphasia took significantly longer than the control group to respond. Participants across the whole group responded significantly more slowly to neutral stimuli. In this case there was no significant interaction between group and valence, but further analysis indicated that the participants with aphasia were quicker at responding to positively and negatively valenced words over words with neutral valence. Control participants responded more quickly to words with positive valence.

\section{Discussion}

Participants with aphasia performed significantly more poorly than the neurotypical controls, in line with previous studies using lexical decision (e.g. Moreno, Buchanan \& Van Orden, 2002). Key differences in response to emotional valence were observed between those with and without aphasia, though there appears to be a facilitatory influence of positively valenced stimuli for both groups.

For the neurotypical control participants emotionally valenced words only showed a processing advantage in terms of response time and only positively valenced words provoked a significantly quicker response. This contrasts with previous research with neurotypical adults (Kousta et al., 2009), though this may be the result of marked differences in the ages of participants in the two studies (mean age in this study: 61.5; in Kousta et al (2009): 19.15 years).

For people with aphasia, a processing advantage was observed for emotionally valenced words over neutral words. These findings mirror previous research in word recognition in aphasia (e.g. Landis, 2006). However previous studies have not considered the effects of whether words were positively or negatively valenced. The findings of this study suggest words with positive valence may be more 
accurately processed than those with negative valence, indicating the importance of categorising words by polarity.

These findings indicate that emotional valence is an important psycholinguistic variable that should be considered when designing assessment and intervention programmes for individuals with aphasia.

\section{References}

Kertesz, A. (2006). The Western Aphasia Battery - Revised. San Antonia, TX: Pearson.

Kousta, S. T., Vinson, D. P. \& Vigliocco, G. (2009). Emotion words, regardless of polarity, have a processing advantage over neutral words. Cognition, 112, 473-481.

Landis, T. (2006). Emotional words: what's so different from just words?. Cortex, 42, 823-830.

Moreno, M. A., Buchanan, L. \& Van Orden, G. C. (2002). Variability in aphasic patients' response times. Brain \& Cognition, 48, 469-474. 